\title{
Weighted Lomax distribution
}

\author{
N. M. Kilany*
}

${ }^{*}$ Correspondence:

neveenkilany@hotmail.com Faculty of Science, Menoufia University, Shebin El-Kom, Egypt

\begin{abstract}
The Lomax distribution (Pareto Type-II) is widely applicable in reliability and life testing problems in engineering as well as in survival analysis as an alternative distribution. In this paper, Weighted Lomax distribution is proposed and studied. The density function and its behavior, moments, hazard and survival functions, mean residual life and reversed failure rate, extreme values distributions and order statistics are derived and studied. The parameters of this distribution are estimated by the method of moments and the maximum likelihood estimation method and the observed information matrix is derived. Moreover, simulation schemes are derived. Finally, an application of the model to a real data set is presented and compared with some other well-known distributions.
\end{abstract}

Keywords: Lomax distribution, Weighted distribution, Reliability measures, Method of moments estimation, Maximum likelihood estimation method

\section{Introduction}

Weighted distribution theory gives a unified approach to dealing with model specification and data interpretation problems. Weighted distributions occur frequently in studies related to reliability, survival analysis, analysis of family data, biomedicine, ecology and several other areas, see Stene (1981) and Oluyede and George (2002). Many authors have presented important results on weighted distributions, Rao (1965) introduced a unified concept of weighted distribution and identified various sampling situations that can modeled by weighted distributions. These situations occur when the recorded observations can not be considered as a random sample from the original distributions. This mean that sometimes it is not possible to work with a truly random sample from population of interest. Zelen (1974) introduced weighted distribution to represent what broadly perceived as a length-biased sampling. Patil and Ord (1976) studied a size biased sampling and related invariant weighted distributions. Statistical applications of weighted distributions related to human population and ecology can be found in Patil and Rao (1978). Gupta and Tripathi (1996) studied the weighted version of the bivariate logarithmic series distribution, which has applications in many fields such as: ecology, social and behavioral sciences.

To present the concept of a weighted distribution, suppose that $X$ is a nonnegative random variable with its probability density function (p.d.f.) $f(x)$, then the p.d.f. of the weight random variable $X_{w}$ is given by

$$
f_{w}(x)=\frac{w(x) f(x)}{E(w(x))}, \quad x \geq 0
$$

(0) The Author(s) 2016. This article is distributed under the terms of the Creative Commons Attribution 4.0 International License (http://creativecommons.org/licenses/by/4.0/), which permits unrestricted use, distribution, and reproduction in any medium, provided you give appropriate credit to the original author(s) and the source, provide a link to the Creative Commons license, and indicate if changes were made. 
where $w(x)$ is a nonnegative weight function and $E(w(x))=\int_{0}^{\infty} w(x) f(x) d x$, $0<E(w(x))<\infty$. The random variable $X_{w}$ is called the weight version of $X$ and its distribution is related to $X$ and is called the weighted distribution with weight function $w(x)$. Note that, the weight function $w(x)$ in Eq. (1) gave different practical examples: such as; when $w(x)=x^{\beta}, \beta>0$, then the resulting distribution is called a size biased version of $X$ and the p.d.f. of a size random variable $X_{s}$ is defined as

$$
f_{s}(x)=\frac{x^{\beta} f(x)}{E\left(x^{\beta}\right)}, \quad x \geq 0, \quad \alpha>0
$$

In Eq. (2) when $\beta=1$, then the weight function $w(x)=x$ and the resulting distribution is called a length-biased distribution and the p.d.f. of a length biased random variable $X_{L}$ is taken the following form:

$$
f_{L}(x)=\frac{x f(x)}{E(x)}, \quad x \geq 0, \quad \alpha>0
$$

where $E(X)=\mu$ is the mean of the original distribution [to know more details about different forms of a weight function, see Rao (1985) and Hewa (2011)]. When an investigator records an observation by nature according to certain stochastic model, the recorded observation will not have the original distribution unless every observation is given an equal chance of being recorded. For example, suppose that the original observation $x_{0}$ comes from a distribution with p.d.f $f_{0}\left(x_{0} ; \theta_{1}\right)$, where $\theta_{1}$ is a parameter vector, and that observation $x$ is recording according to re-weighted by weighted function $w\left(x ; \theta_{2}\right)>0, \theta_{2}$ is a new parameter vector, then $x$ comes from a distribution with p.d.f.

$$
f(x)=A w\left(x ; \theta_{2}\right) f_{0}\left(x_{0} ; \theta_{1}\right)
$$

where A is a normalized constant.

The main aim of this paper is to provide another extension of the Lomax distribution. So, the Weighted-Lomax ("WLx" for short) distribution is proposed to offer a more flexible model for modelling data in several areas such as lifetime analysis, engineering and biomedical sciences. The objectives of the research are to study some structural properties of the proposed distribution. Lomax (1987) proposed Lomax distribution (Pareto Type-II distribution), and used it for the analysis of the business failure lifetime data. Lomax distribution often used in business, economics, and actuarial modeling. It is essentially a Pareto distribution that has been shifted so that its support begins at zero. A random variable $X$ is said to be distributed as Lomax with two parameters $\alpha$ (shape parameter) and $\lambda$ (scale parameter) if it has p.d.f.,

$$
f_{L}(x ; \alpha, \lambda)=\frac{\alpha \lambda^{\alpha}}{(y+\lambda)^{\alpha+1}}, \quad x \geq 0, \quad \alpha, \quad \lambda>0
$$

The corresponding cumulative distribution function (c.d.f.) given by

$$
F_{L}(x ; \alpha, \lambda)=1-\left[1+\left(\frac{x}{\lambda}\right)\right]^{-\alpha}
$$


The trend of parameter(s) induction to the baseline distribution has received increased attention in recent years to explore properties and for efficient estimation of the parameters. In the literature, some extensions of Lomax distribution are available such as the exponentiated Lomax by Abdul-Moniem and Abdel-Hameed (2012), Marshall-Olkin extended-Lomax by Ghitany et al. (2007), Gupta et al. (2010), Beta-Lomax (BL), Kumaraswamy Lomax, McDonald-Lomax by Lemonte and Cordeiro (2013), Gamma-Lomax by Cordeiro et al. (2015), the generalized transmuted Lomax by Nofal et al. (2016) and the transmuted Weibull Lomax by Afify et al. (2015).

In this paper, the WLx distribution is proposed with p.d.f.

$$
f(x)=A x^{\beta-1} f_{L}(x ; \alpha, \lambda)
$$

where $\mathrm{A}$ is a normalized constant and $f_{L}(x ; \alpha, \lambda)$ is the p.d.f. of Lomax distribution. Using Eq. (3), the WLx considered in this paper has p.d.f.

$$
f(x)=\frac{\Gamma(\alpha+1) \lambda^{1+\alpha-\beta}}{\Gamma(1+\alpha-\beta) \Gamma(\beta)}\left(\frac{x^{\beta-1}}{(x+\lambda)^{\alpha+1}}\right), \quad x \geq 0, \quad \lambda>0, \quad \alpha>0, \quad 0<\beta<\alpha+1
$$

where $\Gamma(\cdot)$ is the complete gamma function. Note that, when $\beta=1$, the weighted Lomax distribution reduces to the Lomax distribution. The corresponding c.d.f. is

$$
F(x)=\frac{\Gamma(\alpha+1) \lambda^{-\beta} x^{\beta} \times{ }_{2} F_{1}\left(\alpha+1, \beta, \beta+1 ;-\frac{x}{\lambda}\right)}{\beta \Gamma(\beta) \Gamma(1+\alpha-\beta)}
$$

where ${ }_{2} \mathrm{~F}_{1}(a, b, c ; z)$ is the hypergeometric function.

This paper is organized as follows: In "Distributional properties" section the distributional properties of the proposed distribution are derived and studied. Section "Parameter estimation" discusses the estimation problem using the method of moment and the maximum likelihood estimates of the model parameters. The order statistics and the limiting distribution of the extreme values are derived in "Order statistics and extreme values" section. Section "Simulation study" includes the simulation study for WLx distribution. Finally, a real life application is illustrated in "Conclusion" section.

\section{Distributional properties}

\section{Shapes of probability density function}

The behavior of p.d.f. of the WLx distribution $f(x)$ at $x=0$ and $x=\infty$, respectively, is given by

$$
f(0)=\left\{\begin{array}{ll}
\infty, & \text { if } \beta<1 \\
\frac{\alpha}{\lambda}, & \text { if } \beta=1, \\
0, & \text { if } \beta>1
\end{array} \quad f(\infty)=0\right.
$$

The following theorem describes the shape of p.d.f. of the WLx distribution.

\section{Theorem 1 The p.d.f. $f(x)$ of the WLx distribution is}

(i) Decreasing if $\{0\langle\beta \leq 1, \alpha\rangle 0, \lambda>0\}$.

(ii) Unimodal if $\{1\langle\beta\langle\alpha+1, \alpha\rangle 0, \lambda\rangle 0\}$. 
Proof The first derivative of $f(x)$ is given by

$$
f^{\prime}(x)=\frac{g(x)}{x(x+\lambda)} f(x)
$$

where

$$
g(x)=(-2-\alpha+\beta) x+(\beta-1) \lambda
$$

(i) If $\beta=1$, then $g(x)=-(\alpha+1) x<0$. Hence $f^{\prime}(x)<0$ which implies that $f(x)$ is decreasing. If $\beta<1$, then $g(x)<0$ for all $\alpha+1\langle\beta, \lambda\rangle 0$. Hence $f(x)$ is decreasing.

(ii) If $\{1\langle\beta\langle\alpha+1, \alpha\rangle 0, \lambda\rangle 0\}, f^{\prime}(x)=0$ iff $g(x)=0$ which occurs at the point

$$
x_{0}=\frac{(\beta-1) \lambda}{2+\alpha-\beta}
$$

Since

$$
f^{\prime \prime}(x)=\frac{-(2+\alpha-\beta)}{x_{0}\left(x_{0}+\lambda\right)} f\left(x_{0}\right)<0,
$$

Hence $f(x)$ has a local maximum at $x_{0}$. The behavior of WLx distribution density can be illustrated as in the Fig. 1 .

\section{Hazard rate function}

From (5) and (6), the survival and the hazard (or failure) rate functions are obtained by

$$
\begin{aligned}
& S(x)=1-F(x)=1-\frac{\Gamma(\alpha+1) \lambda^{-\beta} x^{\beta} \times{ }_{2} F_{1}\left(\alpha+1, \beta, \beta+1 ;-\frac{x}{\lambda}\right)}{\beta \Gamma(\beta) \Gamma(1+\alpha-\beta)} \\
& h(x)=\frac{f(x)}{S(x)}=\frac{\Gamma(\alpha+1) \beta \lambda^{\alpha+1} x^{\beta-1}(x+\lambda)^{-\alpha-1}}{\beta \lambda^{\beta} \Gamma(1+\alpha-\beta) \Gamma(\beta)-x^{\beta} \Gamma(1+\alpha) \times{ }_{2} F_{1}\left(\alpha+1, \beta, \beta+1 ;-\frac{x}{\lambda}\right)}
\end{aligned}
$$

According to Glaser (1980), in order to study the behavior of $h(x)$, the behavior of $\eta(x)$ is examined where $\eta(x)=-\frac{d}{d x} \ln f(x)$. The following theorem shows the shapes of the hazard rate function of the WLx distribution.

\section{Theorem 2 Hazard rate function of the WLx distribution is}

(i) Decreasing if $\{0\langle\beta \leq 1, \alpha\rangle 0, \lambda>0\}$.

(ii) Upside-down shape if $\{1\langle\beta\langle\alpha+1, \alpha\rangle 0, \lambda\rangle 0\}$.

Proof Since

$$
\eta(x)=-\frac{d}{d x} \ln f(x)=\frac{1-\beta}{x}+\frac{\alpha+1}{x+\lambda}
$$

It follows that

$$
\eta^{\prime}(x)=-\frac{1-\beta}{x^{2}}-\frac{\alpha+1}{(x+\lambda)^{2}}, \quad \eta^{\prime \prime}(x)=\frac{2(1-\beta)}{x^{3}}+\frac{2(\alpha+1)}{(x+\lambda)^{3}}
$$

(i) For $0<\beta \leq 1, \eta^{\prime}(x)<0$ for all $\alpha+1\langle\beta, \lambda\rangle 0$, i.e. $\eta(x)$ is decreasing. Hence, $h(x)$ is also decreasing.

(ii) For $1<\beta<\alpha+1, \eta^{\prime}(x)=0$ occurs at two points 

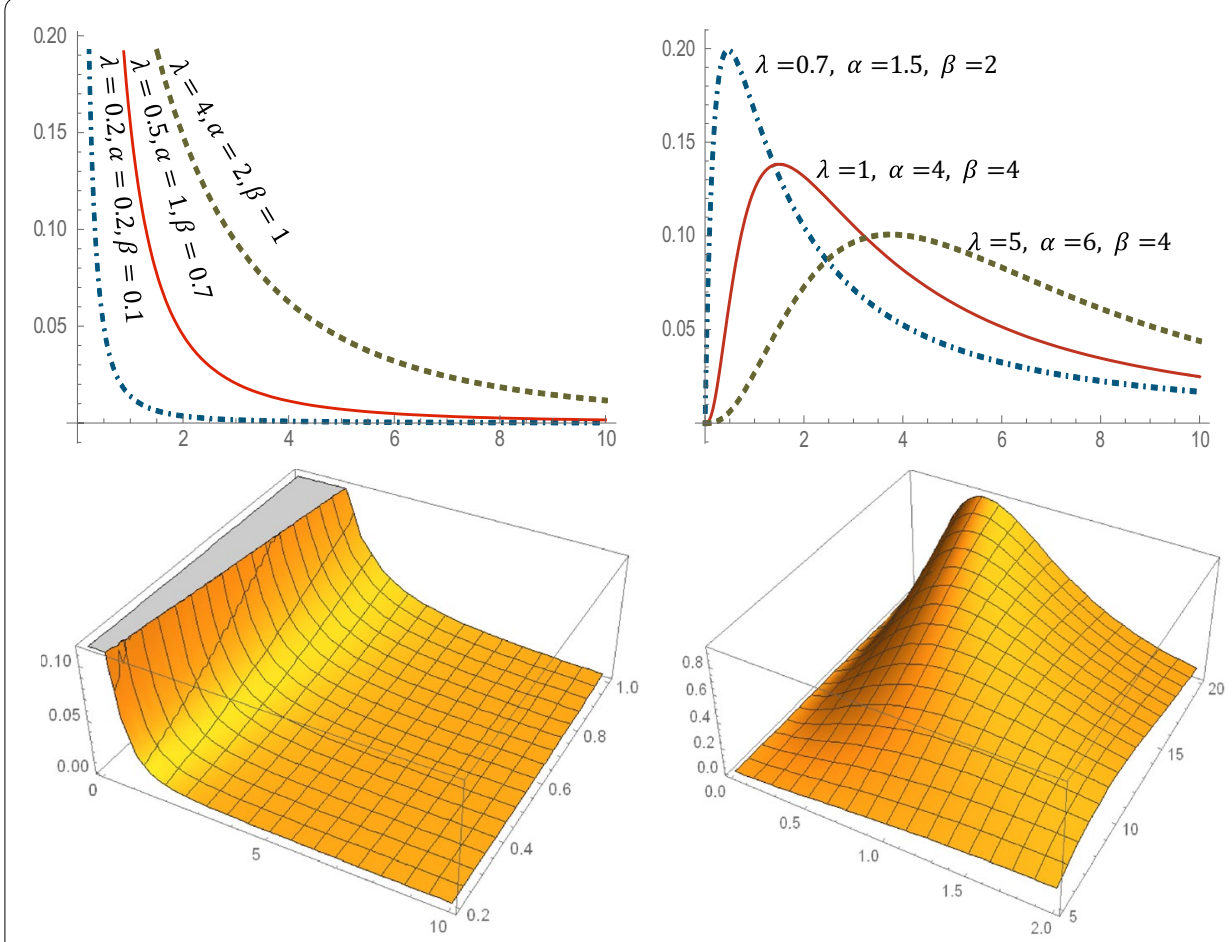

$\lambda=0.5, \alpha=1,0.2<\beta<1$

$\lambda=5, \beta=3,5<\alpha<20$

Fig. 1 Plots of the WLx p.d.f. for some parameter values

$$
x_{1}=\frac{-\lambda(\beta-1)+\lambda \sqrt{(\beta-1)(\alpha+1)}}{-2-\alpha+\beta}, \quad x_{2}=\frac{-\lambda(\beta-1)-\lambda \sqrt{(\beta-1)(\alpha+1)}}{-2-\alpha+\beta}
$$

Since,

$$
\eta^{\prime \prime}\left(x_{2}\right)=-\frac{2(\alpha+1)(2+\alpha-\beta)^{4}(\beta-1)(\sqrt{\beta-1}-\sqrt{\alpha+1})^{2}}{\lambda^{3}\left(\sqrt{(\beta-1)(\alpha+1)}(\sqrt{\beta-1}+\sqrt{\alpha+1})^{2}\right)^{3}}<0,
$$

Then $\eta(x)$ has a local minimum at $x_{2}$ and therefore the hazard function has a local minimum at $x_{2}$ then upside-down shaped. Plots of the WLx hazard function at different parameter values are displayed in Fig. 2.

\section{Mean residual life function and reversed failure rate}

In life testing situations, the remaining lifetimes of a unit of age $x \geq 0$ until the time of failure is known as the residual life. In other words, if $X$ is the life of a unit, then the conditional random variable $[(X-x) / X>x]$ is called the residual life $(R L)$. Gupta and Gupta (1983) showed that the mean residual life function and also the ratio of any two consecutive moments of residual life characterize the distribution. The mean residual life (MRL) function of WLx distribution is given by: 

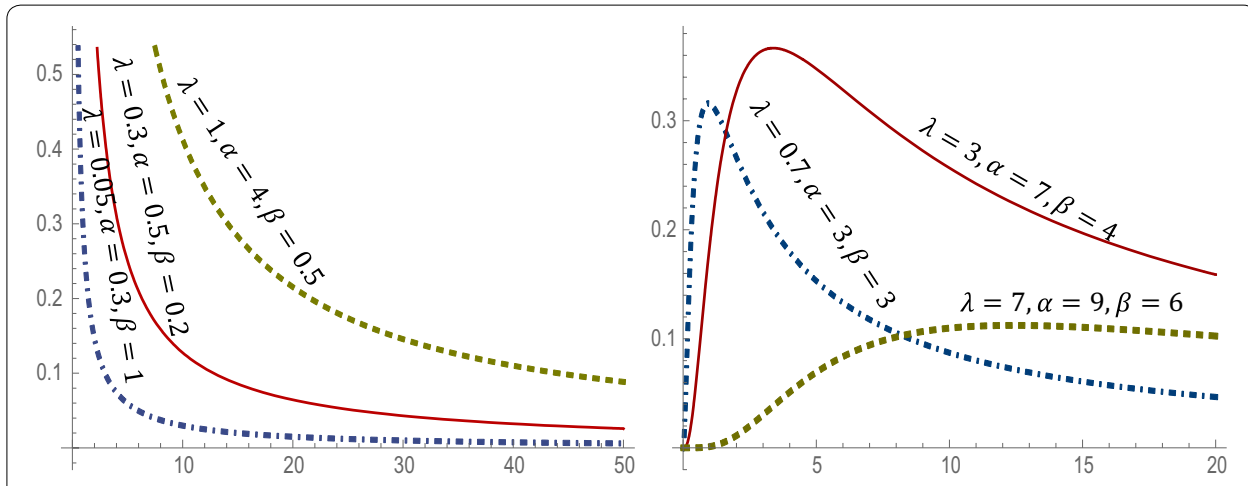

Fig. 2 Plots of the Hazard function of WLX distribution

$$
\begin{aligned}
M R L & =\mu_{1}(x)=E(X-x / X>x)=\frac{1}{S(x)} \int_{x}^{\infty} y f(y) d y-x \\
& =-x+\frac{\beta(-\lambda)^{-\alpha+\beta} \lambda^{\alpha+1} \mathrm{~B}\left(-\frac{\lambda}{x} ; \alpha-\beta,-\alpha\right) \Gamma(\alpha+1)}{\beta \lambda^{\beta} \Gamma(1+\alpha-\beta) \Gamma(\beta)-x^{\beta} \Gamma(\alpha+1) \times_{2} F_{1}\left(\alpha+1, \beta, \beta+1 ;-\frac{x}{\lambda}\right)}
\end{aligned}
$$

where $\mathrm{B}(x ; a, b)$ is the incomplete beta function. The following two Lemmas are useful to determining the shape of mean residual life function $\mu(x)$.

Lemma 2 (Bryson and Siddique 1969) Let $X$ be a non-negative continuous random variable with hazard rate function $h(x)$ and mean residual life function $\mu(x)$. If $h(x)$ is increasing (decreasing), then $\mu(x)$ is increasing (decreasing).

Lemma 3 (Gupta and Akman 1995) Let X be a non-negative continuous random variable with p.d.f. $f(x)$, hazard rate function $h(x)$ and mean residual life function $\mu(x)$. If $h(x)$ has bathtub (upside-down bathtub) shaped and $f(0) \mu(0)>1(=1)$, then $\mu(x)$ has upsidedown bathtub (bathtub) shape.

Using Lemmas 2 and 3, the following theorem shows the shape of the mean residual life function $\mu(x)$ of the WLx distribution.

Theorem 3 The mean residual life function $\mu(x)$ of the WLx distribution is increasing (bathtub shaped) if $0<\beta \leq 1(1<\beta<\alpha+1)$ for all $\alpha>0, \lambda>0$.

Proof Since $h(x)$ is decreasing for $0<\beta \leq 1$, then $\mu(x)$ is increasing. Moreover, since $h(x)$ is upside-down shaped for $1<\beta<\alpha+1$ and $f(0) \mu(0) \leq 0, \mu(x)$ is bathtub shaped. Figure 3 illustrates the plot of mean residual life function of WLx distribution at different parameter values.

The reversed residual life can be defined as the conditional random variable $[x-X / X \leq x]$ which denotes the time elapsed from the failure of a component given that its life is less than or equal to $x, x \geq 0$. This random variable is called also the inactivity time or time since failure. 

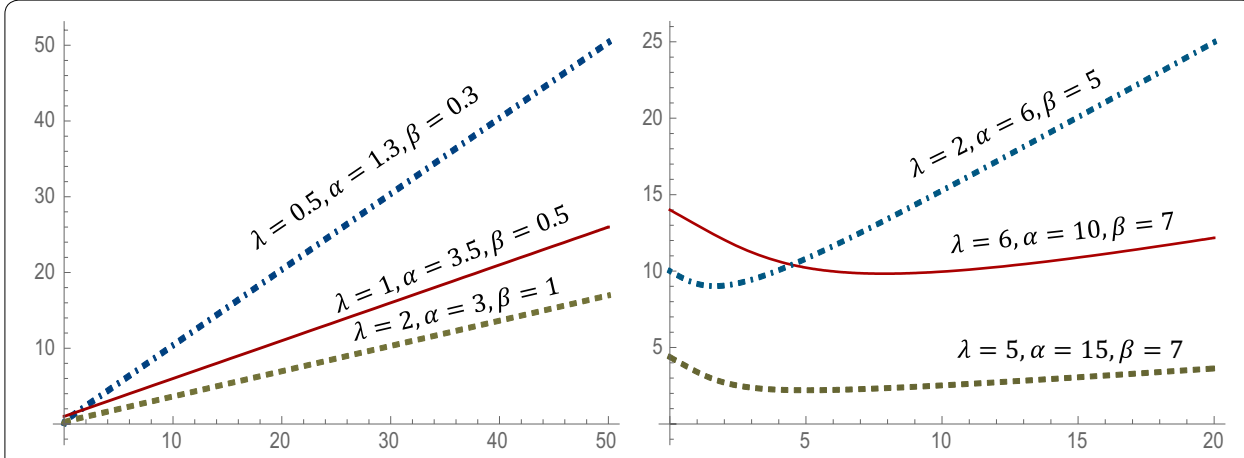

Fig. 3 Plots of the mean residual life of WLx distribution

In addition, in reliability, it is well known that the mean reversed residual life and ratio of two consecutive moments of reversed residual life characterize the distribution uniquely; for more details see Kundu and Nanda (2010) and Nanda et al. (2003). The reversed failure for WLx Distribution is derived as follows:

$$
R h(x)=\frac{f(x)}{F(x)}=\frac{\beta \lambda^{1+\alpha}(x+\lambda)^{-1-\alpha}}{x \times{ }_{2} F_{1}\left(\alpha+1, \beta, \beta+1 ;-\frac{x}{\lambda}\right)}
$$

The rth moment of the reversed residual life is obtained by:

$$
m_{r}(x)=E\left((x-X)^{r} / X \leq x\right)=\frac{1}{F(x)} \int_{0}^{x} r(x-t)^{r-1} F(t) d t
$$

Thus the mean of the reversed residual life is:

$$
m_{1}(x)=\frac{x \times{ }_{2} F_{1}\left(\alpha+1, \beta, \beta+1 ;-\frac{x}{\lambda}\right)}{(\beta+1) \times{ }_{2} F_{1}\left(\alpha+1, \beta, \beta+1 ;-\frac{x}{\lambda}\right)}
$$

The second moment of the reversed residual life is:

$$
m_{2}(x)=\frac{\lambda^{2} \mathrm{~B}\left(-\frac{x}{\lambda} ; 2+\beta,-\alpha\right)}{\mathrm{B}\left(-\frac{x}{\lambda} ; \beta,-\alpha\right)}+x^{2}\left(-1+\frac{2 \times{ }_{2} \tilde{F}_{1}\left(1+\alpha, \beta, 2+\beta,-\frac{x}{\lambda}\right)}{{ }_{2} \tilde{F}_{1}\left(1+\alpha, \beta, 1+\beta,-\frac{x}{\lambda}\right)}\right)
$$

where, ${ }_{2} \tilde{F}_{1}(a, b, c ; z)=\frac{{ }_{2} F_{1}(a, b, c ; z)}{\Gamma(c)}$ is the regularized hypergeometric function.

\section{Moments and associated measures}

The first four moments about the origin of WLx distribution

$$
\begin{aligned}
& \mu_{1}^{\prime}=\frac{\beta \lambda}{\alpha-\beta} \\
& \mu_{2}^{\prime}=\frac{\beta(\beta+1) \lambda^{2}}{(-1+\alpha-\beta)(\alpha-\beta)} \\
& \mu_{3}^{\prime}=\frac{\beta(\beta+1)(\beta+2) \lambda^{3}}{(-2+\alpha-\beta)(-1+\alpha-\beta)(\alpha-\beta)}
\end{aligned}
$$




$$
\mu_{4}^{\prime}=\frac{\lambda^{4} \Gamma(-3+\alpha-\beta) \Gamma(\beta+4)}{\Gamma(1+\alpha-\beta) \Gamma(\beta)}
$$

The central moments about the mean are given by

$$
\begin{aligned}
& \mu_{2}=\frac{\alpha \beta \lambda^{2}}{(-1+\alpha-\beta)(\alpha-\beta)^{2}}, \\
& \mu_{3}=\frac{2 \alpha \beta(\alpha+\beta) \lambda^{3}}{(-2+\alpha-\beta)(-1+\alpha-\beta)(\alpha-\beta)^{3}}, \\
& \mu_{4}=\frac{3 \alpha \beta\left(-\alpha(-2+\beta) \beta+2 \beta^{2}+\alpha^{2}(2+\beta)\right) \lambda^{4}}{(-3+\alpha-\beta)(-2+\alpha-\beta)(-1+\alpha-\beta)(\alpha-\beta)^{4}} .
\end{aligned}
$$

Hence; the mean $(\mu)$ and variance $\left(\sigma^{2}\right)$ of WLx distribution are $\mu=\frac{\beta \lambda}{\alpha-\beta}$ and $\sigma^{2}=\frac{\alpha \beta \lambda^{2}}{(-1+\alpha-\beta)(\alpha-\beta)^{2}}$.

The coefficients of skewness $\left(\beta_{1}\right)$, kurtosis $\left(\beta_{2}\right)$ and variation $(C V)$ of WLx distribution are given by:

$$
\begin{aligned}
& \beta_{1}= \\
& \sqrt{\frac{\left(2 \Gamma(\alpha-\beta)^{3} \Gamma(1+\beta)^{3}-3 \Gamma(-1+\alpha-\beta) \Gamma(\alpha-\beta) \Gamma(1+\alpha-\beta) \Gamma(\beta) \Gamma(1+\beta) \Gamma(2+\beta)+\Gamma(-2+\alpha-\beta) \Gamma(1+\alpha-\beta)^{2} \Gamma(\beta)^{2} \Gamma(3+\beta)\right)^{2}}{\left(-\Gamma(\alpha-\beta)^{2} \Gamma(1+\beta)^{2}+\Gamma(-1+\alpha-\beta) \Gamma(1+\alpha-\beta) \Gamma(\beta) \Gamma(2+\beta)\right)^{3}}} \\
& \beta_{2}=\frac{1}{\left(\Gamma(\alpha-\beta)^{2} \Gamma(1+\beta)^{2}-\Gamma(-1+\alpha-\beta) \Gamma(1+\alpha-\beta) \Gamma(\beta) \Gamma(2+\beta)\right)^{2}} \\
& \times\left(\Gamma ( \alpha - \beta ) \Gamma ( 1 + \beta ) \left[-3 \Gamma(\alpha-\beta)^{3} \Gamma(1+\beta)^{3}\right.\right. \\
& +6 \Gamma(-1+\alpha-\beta) \Gamma(\alpha-\beta) \Gamma(1+\alpha-\beta) \Gamma(\beta) \Gamma(1+\beta) \Gamma(2+\beta) \\
& \left.-4 \Gamma(-2+\alpha-\beta) \Gamma(1+\alpha-\beta)^{2} \Gamma(\beta)^{2} \Gamma(3+\beta)\right] \\
& \left.+\Gamma(-3+\alpha-\beta) \Gamma(1+\alpha-\beta)^{3} \Gamma(\beta)^{3} \Gamma(4+\beta)\right) \\
& C V=\frac{(\alpha-\beta) \sqrt{\frac{\lambda^{2}\left(-\Gamma(1+\beta)^{2}+\frac{(\alpha-\beta) \Gamma(\beta) \Gamma(2+\beta)}{-1+\alpha-\beta}\right)}{(\alpha-\beta)^{2}}}}{\lambda \Gamma(1+\beta)}
\end{aligned}
$$

Moreover, the moment generating $M_{x}(t)$ and characteristic functions $\phi_{x}(t)$ are

$$
\begin{gathered}
M_{x}(t)=\frac{\frac{1+\lambda-t \lambda}{(-1+t \lambda)^{2}}+e^{-t \lambda}(-t)^{\alpha} \alpha \lambda^{\alpha} \lambda(1+\lambda) \Gamma(-\alpha,-t \lambda)}{(1+\lambda)^{2}}, \\
\phi_{x}(t)=\frac{\pi \operatorname{Csc}(\pi(\alpha-\beta))\left(\frac{(-\mathrm{i} t)^{1+\alpha-\beta} \lambda^{1+\alpha-\beta} \Gamma(1+\alpha)_{1} \tilde{F}_{1}(1+\alpha, 2+\alpha-\beta,-\mathrm{i} \lambda t)}{\Gamma[\beta]}-{ }_{1} \tilde{F}_{1}(\beta,-\alpha+\beta ;-\mathrm{i} \lambda t)\right)}{\Gamma(1+\alpha-\beta)(1+\theta)^{2}(\theta-\mathrm{i} t)^{2}},
\end{gathered}
$$

where $\Gamma(a, z)$ is the incomplete gamma function, and $\operatorname{Csc}(z)$ is the cosecant of $z$. 


\section{Lorenz and Bonferroni curves}

The Bonferroni, Lorenz curves and Gini index have many applications in economics and ecology to describe inequality distribution of wealth or size like a reliability, medicine and insurance. For WLx distribution, Lorenz and Bonferroni curves are:

$$
L_{F}[F(x)]=\frac{x^{1+\beta} \lambda^{-1-\beta} \Gamma(1+\alpha)_{2} \tilde{F}_{1}\left(1+\alpha, 1+\beta, 2+\beta ;-\frac{x}{\lambda}\right)}{\Gamma(\alpha-\beta)}
$$

and

$$
B_{F}(F(x))=\frac{(-\alpha+\beta) \mathrm{B}\left(-\frac{x}{\lambda} ; 1+\beta,-\alpha\right)}{\beta \mathrm{B}\left(-\frac{x}{\lambda} ; \beta,-\alpha\right)}
$$

.The scaled total time on test transform of a distribution function is given by

$$
S_{F}[F(t)]=\frac{x\left(\alpha-\beta-\frac{x^{\beta} \lambda^{-\beta} \Gamma(1+\alpha)_{2} \tilde{F}_{1}\left(1+\alpha, \beta, 2+\beta,-\frac{x}{\lambda}\right)}{\Gamma(\alpha-\beta)}\right)}{\beta \lambda}
$$

Hence, Gini index is obtained from the relationship $G=1-C_{F}$; where $C_{F}=\int_{0}^{1} S_{F}[F(t)] f(t) d t$.

\section{Entropies}

An entropy is a measure of variation or uncertainty of a random variable $X$. The theory of entropy has been successfully used in a wide diversity of applications and has been used for the characterization of numerous standard probability distributions. Two popular entropy measures are the Shannon and Rényi entropies.

The Rényi entropy of a random variable with p.d.f. $f(x)$ is defined as

$$
I_{\delta}=\frac{1}{1-\delta} \log \int_{0}^{\infty} f^{\delta}(x) d x, \quad \delta>0, \quad \delta \neq 1
$$

For WLx distribution, Rényi entropy is derived as

$$
\begin{aligned}
I_{\delta}= & \frac{1}{1-\delta} \log \left(\frac{\Gamma(\alpha+1) \lambda^{\alpha-\beta+1}}{\Gamma(\alpha-\beta+1) \Gamma(\beta)}\right)^{\delta} \int_{0}^{\infty}\left(\frac{x^{\beta-1}}{(x+\lambda)^{\alpha+1}}\right)^{\delta} d x \\
= & \log \lambda+\delta(1-\delta)^{-1} \log \left(\frac{\Gamma(\alpha+1)}{\Gamma(\alpha-\beta+1) \Gamma(\beta)}\right) \\
& +(1-\gamma)^{-1} \log \left(\frac{\Gamma(-1+(2-\alpha-\beta) \delta) \Gamma(1+(\beta-1) \delta}{\Gamma(\delta+\alpha \delta)}\right)
\end{aligned}
$$

The Shannon entropy of a random variable $X$ is defined by

$$
S_{H}=-\int_{0}^{\infty} f(x) \log f(x) d x
$$

Using the WLx density,

$$
\begin{aligned}
S_{H}= & -\frac{\Gamma(\alpha+1) \lambda^{\alpha-\beta+1}}{\Gamma(\alpha-\beta+1) \Gamma(\beta)} \int_{0}^{\infty} \frac{x^{\beta-1}}{(x+\lambda)^{\alpha+1}} \log \frac{\Gamma(\alpha+1) \lambda^{1+\alpha-\beta}}{\Gamma(1+\alpha-\beta) \Gamma(\beta)}\left(x^{\beta-1}(x+\lambda)^{-\alpha-1}\right) d x \\
= & -\log \frac{\Gamma(\alpha+1) \lambda^{\alpha-\beta+1}}{\Gamma(\alpha-\beta+1) \Gamma(\beta)}-(\beta-1)(\log \lambda-\psi(\alpha-\beta+1)+\psi(\beta)) \\
& +(\alpha+1)\left(H_{\alpha}-H_{\alpha-\beta}+\log \lambda\right) \\
= & \log \frac{\lambda \Gamma(\alpha-\beta+1) \Gamma(\beta)}{\Gamma(\alpha+1)}+(\alpha+1) H_{\alpha}+(\beta-\alpha-2) H_{\alpha-\beta}-(\beta-1)(\gamma+\psi(\beta))
\end{aligned}
$$


where $H_{n}$ is the $n$-th harmonic number, $\gamma$ is the Euler-Mascheroni constant and $\psi(z)$ is the digamma function.

\section{Reliability parameter}

In the context of reliability, the stress-strength model describes the life of a component that has a random strength $Y$ that is subjected to a random stress $X$. The component fails at the instant that the stress applied to it exceeds the strength, and the component will function satisfactorily whenever $X<Y$. Hence, $R=\operatorname{Pr}(X<Y)$ is a measure of component reliability. It has many applications especially in the areas of engineering and stress-strength models. The reliability parameter for WLx distribution is given by

$$
\begin{aligned}
R= & \operatorname{Pr}(X<Y) \\
= & \frac{\lambda_{1}^{\alpha_{1}-\beta_{1}+1} \Gamma\left(\alpha_{1}+1\right)}{\Gamma\left(\alpha_{1}-\beta_{1}+1\right) \Gamma\left(\beta_{1}\right)} \times \frac{\lambda_{2}^{\alpha_{2}-\beta_{2}+1} \Gamma\left(\alpha_{2}+1\right)}{\Gamma\left(\alpha_{2}-\beta_{2}+1\right) \Gamma\left(\beta_{2}\right)} \times \int_{0}^{\infty} \int_{0}^{y}\left(\frac{x^{\beta_{1}-1}}{\left(x+\lambda_{1}\right)^{\alpha_{1}+1}}\right)\left(\frac{y^{\beta_{2}-1}}{\left(y+\lambda_{2}\right)^{\alpha_{2}+1}}\right) d x d y \\
= & \frac{\lambda_{1}^{-\beta_{1}} \Gamma\left(\alpha_{1}+1\right)}{\Gamma\left(\alpha_{1}-\beta_{1}+1\right)} \times \frac{\lambda_{2}^{\alpha_{2}-\beta_{2}+1} \Gamma\left(\alpha_{2}+1\right)}{\Gamma\left(\alpha_{2}-\beta_{2}+1\right) \Gamma\left(\beta_{2}\right)} \times \int_{0}^{\infty} \frac{y^{\beta_{1}+\beta_{2}-1}}{\left(y+\lambda_{2}\right)^{\alpha_{2}+1}} \hat{F}_{1}\left(\alpha_{1}+1, \beta_{1}, \beta_{1}+1 ;-\frac{y}{\lambda_{1}}\right) d y \\
= & \left\{\left[\frac{\left(\lambda_{2} / \lambda_{1}\right)^{\alpha_{2}-\beta_{2}+1} \Gamma\left(\alpha_{2}+1\right) \Gamma\left(2+\alpha_{1}+\alpha_{2}-\beta_{1}-\beta_{2}\right) \Gamma\left(-1-\alpha_{2}+\beta_{1}+\beta_{2}\right)}{\left(\alpha_{2}-\beta_{2}+1\right) \Gamma\left(\beta_{1}\right) \Gamma\left(\beta_{2}\right)\left(\alpha_{1}-\beta_{1}+1\right) \Gamma\left(\alpha_{2}-\beta_{2}+1\right)}\right.\right. \\
& \left.\times{ }_{p} F_{q}\left(\left\{1+\alpha_{2}, 1+\alpha_{2}-\beta_{2}, 2+\alpha_{1}+\alpha_{2}-\beta_{1}-\beta_{2}\right\},\left\{2+\alpha_{2}-\beta_{2}, 2+\alpha_{2}-\beta_{1}-\beta_{2}\right\} ; \frac{\lambda_{2}}{\lambda_{1}}\right)\right] \\
& +\left[\frac{\left(\lambda_{2} / \lambda_{1}\right)^{\beta_{1}} \Gamma\left(\alpha_{1}+1\right) \Gamma\left(1+\alpha_{2}-\beta_{1}-\beta_{2}\right) \Gamma\left(\beta_{1}+\beta_{2}\right)}{\left(\alpha_{2}-\beta_{2}+1\right) \Gamma\left(\beta_{1}\right) \Gamma\left(\beta_{2}\right)\left(\alpha_{1}-\beta_{1}+1\right) \Gamma\left(\alpha_{2}-\beta_{2}+1\right)}\right. \\
& \left.\left.\times{ }_{p} F_{q}\left(\left\{1+\alpha_{1}, \beta_{1}, \beta_{1}+\beta_{2}\right\},\left\{1+\beta_{1},-\alpha_{2}+\beta_{1}+\beta_{2}\right\} ; \frac{\lambda_{2}}{\lambda_{1}}\right)\right]\right\}
\end{aligned}
$$

where ${ }_{p} F_{q}\left(\left\{a_{1}, a_{2}, \ldots, a_{p}\right\},\left\{b_{1}, b_{2}, \ldots, b_{1}\right\} ; z\right)$ is the generalized hypergeometric function.

\section{Parameter estimation}

In this section, we consider the method of moments and the maximum likelihood techniques is considered to estimate the involved parameters of WLx distribution. In addition, the interval estimation is discussed via Fisher information matrix.

\section{Method of moments estimates}

The method of moment estimation (MME) consists of equating the first three moments of the population (7), (8) and (9) to the corresponding moments of the sample. The system of equations that needed is:

$$
\begin{aligned}
& \frac{\beta \lambda}{\alpha-\beta}=m_{1} \\
& \frac{\beta(\beta+1) \lambda^{2}}{(-1+\alpha-\beta)(\alpha-\beta)}=m_{2} \\
& \frac{\beta(\beta+1)(\beta+2) \lambda^{3}}{(-2+\alpha-\beta)(-1+\alpha-\beta)(\alpha-\beta)}=m_{3}
\end{aligned}
$$


By solving the Eqs. (10), (11) and (12), the parameter estimates are

$$
\begin{aligned}
& \hat{\lambda}=\frac{m_{1} m_{2}^{2}-2 m_{1}^{2} m_{3}+m_{2} m_{3}}{\left(m_{1}^{2}-2 m_{2}\right) m_{2}+m_{1} m_{3}} \\
& \hat{\alpha}=\frac{2\left(m_{1}^{2}-m_{2}\right)\left(m_{1} m_{2}-m_{3}\right)\left(m_{2}^{2}-m_{1} m_{3}\right)}{\left(\left(m_{1}^{2}-2 m_{2}\right) m_{2}+m_{1} m_{3}\right)\left(-m_{1} m_{2}^{2}+\left(2 m_{1}^{2}-m_{2}\right) m_{3}\right)} \\
& \hat{\beta}=\frac{2 m_{1}\left(m_{2}^{2}-m_{1} m_{3}\right)}{-m_{1} m_{2}^{2}+\left(2 m_{1}^{2}-m_{2}\right) m_{3}} .
\end{aligned}
$$

\section{Maximum likelihood estimates}

Let $x_{1}, x_{2}, \ldots, x_{n}$ be a random sample of size $\mathrm{n}$ from the WLx distribution, the maximum likelihood estimates (MLEs) of the parameters are obtained by direct maximization of the log-likelihood function which is given by:

$$
\begin{aligned}
\ln (L(x ; \lambda, \alpha, \beta))= & n \ln [\Gamma(\alpha+1)]+n(\alpha-\beta+1) \ln [\lambda]-n \ln [\Gamma(\alpha-\beta+1)] \\
& -n \ln [\Gamma(\beta)]+(\beta-1) \sum_{i=1}^{n} \ln \left[x_{i}\right]-(\alpha+1) \sum_{i=1}^{n} \ln \left[x_{i}+\lambda\right]
\end{aligned}
$$

It follows that the maximum likelihood estimators (MLEs); $\hat{\lambda}, \hat{\alpha}$ and $\hat{\beta}$ are the simultaneous solutions of the equations:

$$
\begin{aligned}
& \frac{n(1+\alpha-\beta)}{\lambda}-(1+\alpha) \sum_{i=1}^{n} \frac{1}{x_{i}+\lambda}=0 \\
& n \ln [\lambda]+n \psi(\alpha+1)-n \psi(\alpha-\beta+1)-\sum_{i=1}^{n} \ln \left[\lambda+x_{i}\right]=0 \\
& -n \ln [\lambda]+n \psi(\alpha-\beta+1)-n \psi(\beta)+\sum_{i=1}^{n} \ln \left[x_{i}\right]=0
\end{aligned}
$$

For interval estimation of the parameter vector $\Theta=(\lambda, \alpha, \beta)^{T}$; the expected fisher information matrix $\mathbf{I}=\left[I_{i j}\right], i, j=1,2,3$ is derived as follows:

$$
\begin{aligned}
I_{11} & =-E\left[\frac{\partial^{2}}{\partial \lambda^{2}} \ln f(x)\right] \\
& =\frac{\pi \operatorname{Csc}(\pi(\alpha-\beta))\left(\frac{(1+\alpha-\beta)^{2}(2+\alpha-\beta)\left(\frac{1+\alpha}{\beta}\right)^{-\alpha}\left(\frac{-1-\alpha+\beta}{\beta}\right)^{\alpha-\beta} \Gamma(1+\alpha)}{(1+\alpha) \Gamma(\beta)}+\frac{-2+{ }_{2} F_{1}\left[1, \beta,-1-\alpha+\beta ; \frac{1-\alpha+\beta}{\beta}\right]}{\Gamma(-2-\alpha+\beta)}\right)}{\lambda \Gamma(3+\alpha-\beta)} ; \\
I_{22} & =-E\left[\frac{\partial^{2}}{\partial \alpha^{2}} \ln f(x)\right]=-\psi^{\prime}(\alpha+1)+\psi^{\prime}(\alpha-\beta+1) ; \\
I_{33} & =-E\left[\frac{\partial^{2}}{\partial \beta^{2}} \ln f(x)\right]=\psi^{\prime}(\alpha-\beta+1)+\psi^{\prime}(\beta) ; \\
I_{12} & =-E\left[\frac{\partial^{2}}{\partial \lambda \partial \alpha} \ln f(x)\right]=-\frac{\beta}{\lambda(\alpha+1)} ; \\
I_{13} & =-E\left[\frac{\partial^{2}}{\partial \lambda \partial \beta} \ln f(x)\right]=\frac{1}{\lambda} ; \\
I_{23} & =-E\left[\frac{\partial^{2}}{\partial \alpha \partial \beta} \ln f(x)\right]=-\psi^{\prime}(\alpha-\beta+1) ;
\end{aligned}
$$

where $\psi^{\prime}(z)$ is the trigamma function. 
Under regularity conditions, Bahadur (1964) showed that as $n \rightarrow \infty, \sqrt{n}(\hat{\Theta}-\Theta)$ is asymptotically normal 3-variate with (vector) mean zero and covariance matrix $\mathbf{I}^{-1}$. The asymptotic variances and covariance of the elements of $\hat{\Theta}$ are given by:

$$
\begin{aligned}
& V(\hat{\alpha})=\frac{I_{22} I_{33}-I_{23}^{2}}{n \Delta}, \quad V(\hat{\beta})=\frac{I_{11} I_{33}-I_{13}^{2}}{n \Delta}, \\
& V(\hat{\theta})=\frac{I_{11} I_{22}-I_{12}^{2}}{n \Delta}, \quad \operatorname{Cov}(\hat{\alpha}, \hat{\beta})=\frac{I_{13} I_{23}-I_{12} I_{33}}{n \Delta}, \\
& \operatorname{Cov}(\hat{\alpha}, \hat{\theta})=\frac{I_{12} I_{23}-I_{13} I_{22}}{n \Delta}, \quad \operatorname{Cov}(\hat{\beta}, \hat{\theta})=\frac{I_{13} I_{12}-I_{11} I_{23}}{n \Delta} .
\end{aligned}
$$

where $\Delta=\operatorname{det}(\mathbf{I})$. The corresponding asymptotic $100(1-\alpha) \%$ confidence intervals are $\hat{\Theta} \pm c \mathbf{I}^{-1 / 2}$; where $\mathrm{c}$ is the appropriate $z$ critical value.

\section{Order statistics and extreme values}

The distribution of extreme values plays an important role in statistical applications. In this section the probability and cumulative function of order statistics are introduced and the limiting distribution minimum and the maximum arising from the WLx model can be derived.

\section{Probability and cumulative function of order statistics}

Suppose $X_{1}, X_{2}, \ldots, X_{n}$ is a random sample from WLx distribution. Let $X_{1: n}<X_{2: n}<\cdots$ $<X_{n: n}$ denote the corresponding order statistics. The probability density function and the cumulative distribution function of the $j$ th order statistic, say $Y=X_{j: n}$, are given by

$$
\begin{aligned}
f_{Y}(y)= & \frac{n !}{(j-1) !(n-j) !} F^{j-1}(y)\{1-F(y)\}^{n-j} f(y) \\
= & \frac{\lambda^{1+\alpha}(y+\lambda)^{-1-\alpha} n !}{y \Gamma(j) \Gamma(1-j+n) \Gamma(\beta)_{2} \tilde{F}_{1}\left(1+\alpha, \beta, 1+\beta ;-\frac{y}{\lambda}\right)} \\
& \times\left[\frac{y^{\beta} \lambda^{-\beta} \Gamma(1+\alpha)_{2} \tilde{F}_{1}\left(1+\alpha, \beta, 1+\beta ;-\frac{y}{\lambda}\right)}{\Gamma(1+\alpha-\beta)}\right]^{j} \\
& \times\left[1-\frac{y^{\beta} \lambda^{-\beta} \Gamma(1+\alpha)_{2} \tilde{F}_{1}\left(1+\alpha, \beta, 1+\beta ;-\frac{y}{\lambda}\right)}{\Gamma(1+\alpha-\beta)}\right]^{n-j}
\end{aligned}
$$

and

$$
\begin{aligned}
F_{Y}(y)= & \sum_{m=j}^{n}\left(\begin{array}{c}
n \\
m
\end{array}\right) \mathrm{F}^{m}(y) \times[1-\mathrm{F}(\mathrm{y})]^{n-m} \\
= & \frac{\Gamma(1+n)_{2} \tilde{F}_{1}\left(1, j-n,+j ; \frac{1}{1-\frac{y^{-\beta} \lambda^{\beta}(1+\alpha-\beta)}{\Gamma(1+\alpha) \tilde{F}_{1}\left(1+\alpha, \beta, 1+\beta ;-\frac{y}{\lambda}\right)}}\right)}{\Gamma(1-j+n)} \\
& \times\left[\frac{y^{\beta} \lambda^{-\beta} \Gamma(1+\alpha)_{2} \tilde{F}\left(1+\alpha, \beta, 1+\beta ;-\frac{y}{\lambda}\right)}{\Gamma(1+\alpha-\beta)}\right]^{j} \\
& \times\left[1-\frac{y^{\beta} \lambda^{-\beta} \Gamma(1+\alpha)_{2} \tilde{F}\left(1+\alpha, \beta, 1+\beta,-\frac{y}{\lambda}\right)}{\Gamma(1+\alpha-\beta)}\right]^{n-j}
\end{aligned}
$$




\section{Limiting distributions of extreme values}

Let $m_{n}=X_{1: n}=\min \left[X_{1}, \mathrm{X}_{2}, \ldots, \mathrm{X}_{n}\right]$ and $M_{n}=X_{n: n}=\max \left[X_{1}, \mathrm{X}_{2}, \ldots, \mathrm{X}_{n}\right]$ arising from WLx distribution. The limiting distributions of $X_{1: n}$ and $X_{n: n}$ can be derived in the following theorem.

Theorem 3 Let $m_{n}$ and $M_{n}$ be the minimum and the maximum of a random sample from the WLx distribution, respectively. Then

(i) $\lim _{n \rightarrow \infty} p\left(\frac{m_{n}-a_{n}}{b_{n}} \leq x\right)=1-\exp \left(-x^{\beta}\right) ; \quad x>0$

(ii) $\lim _{n \rightarrow \infty} p\left(\frac{M_{n}-c_{n}}{d_{n}} \leq x\right)=\exp \left(-x^{-(1+\alpha-\beta)}\right) ; \quad x>0$ where $a_{n}=0, b_{n}=\frac{1}{F^{-1}\left(\frac{1}{n}\right)}, c_{n}=0$ and $d_{n}=\frac{1}{F^{-1}\left(1-\frac{1}{n}\right)}$.

Proof (i) Using L'Hospital rule, we have

$$
\lim _{\varepsilon \rightarrow 0^{+}} \frac{F\left(F^{-1}(0)+\varepsilon x\right)}{F\left(F^{-1}(0)+\varepsilon\right)}=\lim _{\varepsilon \rightarrow 0^{+}} \frac{F(\varepsilon x)}{F(\varepsilon)}=\lim _{\varepsilon \rightarrow 0^{+}} \frac{x f(\varepsilon x)}{f(\varepsilon)}=x^{\beta} .
$$

Therefore by Theorem (8.3.6) of Arnold et al. (1992), the minimal domain of attraction of the WLx distribution is the Weibull distribution, proving part (i).

(ii) Using L'Hospital rule, we have

$$
\lim _{t \rightarrow \infty} \frac{1-F(t x)}{1-F(t)}=\lim _{t \rightarrow \infty} \frac{x f(t x)}{f(t)}=x^{-(1+\alpha-\beta)}
$$

Therefore, by Theorem (1.6.2) and Corollary (1.6.3) in Leadbetter et al. (1987), the maximal domain of attraction of the WLx distribution is the Fréchet distribution, proving part (ii).

\section{Simulation study}

The equation $F(x)-u=0$, where $u$ is an observation from the uniform distribution $(0,1)$ and $F(x)$ is cumulative distribution function of WLx distribution, is used to carry out the simulation study by generating random samples follow WLx distribution. The simulation experiment was repeated 1000 times each with sample sizes; 20, 40, 70,100 for $(\lambda, \alpha, \beta)=(0.03,2,0.5)$ and $(0.01,2.5,1)$. The following measures are computed:

(i) Average bias of $\hat{\lambda}, \hat{\alpha}$ and $\hat{\beta}$ of the parameters $\lambda, \alpha$ and $\beta$ are respectively;

$$
\frac{1}{N} \sum_{i=1}^{N}(\hat{\lambda}-\lambda), \quad \frac{1}{N} \sum_{i=1}^{N}(\hat{\alpha}-\alpha) \quad \text { and } \quad \frac{1}{N} \sum_{i=1}^{N}(\hat{\beta}-\beta)
$$

(ii) The Mean square error (MSE) of $\hat{\lambda}, \hat{\alpha}$ and $\hat{\beta}$ of the parameters $\lambda, \alpha$ and $\beta$ are respectively;

$$
\frac{1}{N} \sum_{i=1}^{N}(\hat{\lambda}-\lambda)^{2}, \quad \frac{1}{N} \sum_{i=1}^{N}(\hat{\alpha}-\alpha)^{2} \quad \text { and } \quad \frac{1}{N} \sum_{i=1}^{N}(\hat{\beta}-\beta)^{2}
$$

Table 1 presents the average bias and the MSE of the estimates. The values of the bias are seen to be small,positive and the values of the MSEs decreases while the sample size increases. 
Table 1 Bias and MSE for the parameters $\lambda, \alpha, \beta$

\begin{tabular}{llllllllll}
\hline $\boldsymbol{\lambda}$ & $\boldsymbol{a}$ & $\boldsymbol{\beta}$ & $\mathbf{n}$ & $\operatorname{Bias}(\boldsymbol{\lambda})$ & $\operatorname{MSE}(\boldsymbol{\lambda})$ & $\operatorname{Bias}(\boldsymbol{\alpha})$ & $\operatorname{MSE}(\boldsymbol{\alpha})$ & $\operatorname{Bias}(\boldsymbol{\beta})$ & $\operatorname{MSE}(\boldsymbol{\beta})$ \\
\hline 0.03 & 2 & 0.5 & 20 & -0.0285 & 0.00299 & 4.6345 & 35.5596 & 4.7126 & 36.5100 \\
& & & 40 & -0.0266 & 0.00071 & 3.8839 & 23.5742 & 4.0339 & 24.7811 \\
& & & 70 & -0.0261 & 0.00068 & 3.1262 & 15.2725 & 3.3071 & 16.3899 \\
& & & 100 & -0.0257 & 0.00066 & 2.7709 & 11.9247 & 2.9629 & 12.9699 \\
0.01 & 2.5 & 1 & 20 & -0.0083 & 0.000069 & 5.8120 & 60.4177 & 5.8464 & 61.0917 \\
& & & 40 & -0.0081 & 0.000066 & 5.1198 & 45.5128 & 5.2032 & 46.4064 \\
& & & 70 & -0.0078 & 0.000061 & 4.0102 & 28.6942 & 4.1131 & 29.5265 \\
& & & 100 & -0.0075 & 0.000058 & 3.4679 & 22.5719 & 3.5759 & 23.317 \\
\hline
\end{tabular}

\section{Application}

The considered a dataset corresponding to remission times (in months) of a random sample of 128 bladder cancer patients given in Lee and Wang (2003). The data are given as follows: $0.08,2.09,3.48,4.87,6.94,8.66,13.11,23.63,0.20,2.23,3.52,4.98,6.97,9.02$, $13.29,0.40,2.26,3.57,5.06,7.09,9.22,13.80,25.74,0.50,2.46,3.64,5.09,7.26,9.47$, $14.24,25.82,0.51,2.54,3.70,5.17,7.28,9.74,14.76,26.31,0.81,2.62,3.82,5.32,7.32$, $10.06,14.77,32.15,2.64,3.88,5.32,7.39,10.34,14.83,34.26,0.90,2.69,4.18,5.34,7.59$, $10.66,15.96,36.66,1.05,2.69,4.23,5.41,7.62,10.75,16.62,43.01,1.19,2.75,4.26,5.41$, 7.63, 17.12, 46.12, 1.26, 2.83, 4.33, 5.49, 7.66, 11.25, 17.14, 79.05, 1.35, 2.87, 5.62, 7.87, $11.64,17.36,1.40,3.02,4.34,5.71,7.93,11.79,18.10,1.46,4.40,5.85,8.26,11.98,19.13$, $1.76,3.25,4.50,6.25,8.37,12.02,2.02,3.31,4.51,6.54,8.53,12.03,20.28,2.02,3.36,6.76$, $12.07,21.73,2.07,3.36,6.93,8.65,12.63,22.69$. We have fitted the WLx distribution to the dataset using MLE, and compared the proposed WLx distribution with, gammaLomax (GL), Kumaraswamy-Lomax(KwL), transmuted exponentiated-Lomax (TrEL), Weibull-Lomax (WL), McDonald-Lomax (McL), Beta-Lomax(BL), extended PoissonLomax (EPL), exponential-Lomax (ExL) and Lomax distributions.

The c.d.f.(s) of these models are given as follow:

- The McLomax (McL) density function with five parameters $\alpha, \lambda, \beta, a$ and $b$ introduced by Lemonte and Cordeiro (2013) is expressed as

$$
f_{M c L}(x)=\frac{\beta \alpha \lambda^{\alpha}(\lambda+x)^{-(\alpha+1)}}{B\left(a \beta^{-1}, b+1\right)}\left(1-\left(\frac{\lambda}{\lambda+x}\right)^{\alpha}\right)^{a-1}\left(1-\left(1-\left(\frac{\lambda}{\lambda+x}\right)^{\alpha}\right)^{\beta}\right)^{b},
$$

(where $x>0 ; \alpha, \lambda, \beta, b, a,>0$ ).

Evidently, the McL density function does not involve any complicated function, and it includes several distributions as special sub-models not previously considered in the literature. In fact, the Lomax distribution (with parameters $\alpha$ and $\lambda$ ) is clearly a basic exemplar for $a=\beta=1$ and $b=0$. Beta Lomax (BL) and Kumaraswamy Lomax $(\mathrm{KwL})$ distributions are new models which arise for $\beta=1$ and $a=\beta$, respectively. For $b=0$ and $\beta=1$, it leads to a new distribution referred to as the Exponentiated Lomax (EL) distribution. The McL distribution allows for greater flexibility of its tails and can be widely applied in many areas. The c.d.f. corresponding to McL density function is given by

$$
F_{M c L}(x)=I_{\left\{1-\lambda^{\alpha}(\lambda+x)^{-\alpha}\right\}^{\beta}}\left(a \beta^{-1}, b+1\right)
$$


- The Exponential Lomax (ExL) distribution introduced by El-Bassiouny et al. (2015) with c.d.f.

$$
F_{E L}(x)=1-\mathrm{e}^{-\beta \times\left(\frac{\lambda}{x+\lambda}\right)^{-\alpha}}, \quad x \geq-\lambda, \quad \alpha, \quad \lambda, \quad \beta>0
$$

- The gamma-Lomax (GL) distribution introduced by Cordeiro et al. (2015) based on a versatile and flexible gamma generator proposed by Zagrafos and Balakrishnan (2009) using Stacy's generalized gamma distribution and record value theory. The c.d.f. of GL distribution is given by

$$
F_{G L}(x)=\frac{\Gamma\left[a, \alpha \log \left[1+\frac{x}{\lambda}\right]\right]}{\Gamma[a]}, \quad x>0, \quad \alpha, \lambda, a>0
$$

- The transmuted Exponentiated-Lomax (TrEL) distribution introduced by Ashour and Eltehiwy (2013) with c.d.f.

$$
F(x)=\left(1-(1+\lambda x)^{-\alpha}\right)^{\beta}\left((1+\gamma)-\gamma\left(1-(1+\lambda x)^{-\alpha}\right)^{\beta}\right),
$$

(where $>0 ; \alpha, \lambda, \beta, \gamma>0$ )

- The Weibull-Lomax (WL) distribution introduced by Tahir et al. (2015) with c.d.f.

$$
F_{W L}(x)=1-\mathrm{e}^{\left(-a\left(\left(1+\left(\frac{x}{\lambda}\right)\right)^{\alpha}-1\right)^{b}\right)}, \quad x>0, \quad a, b, \alpha, \lambda>0
$$

- The extended Poisson-Lomax (EPL) distribution introduced by Al-Zahrani et al. (2015) introduced with c.d.f.

$$
F_{E P L}(x)=1-(1+\lambda x)^{-\alpha} \mathrm{e}^{-\beta\left(1-(1+\lambda x)^{-\alpha}\right)}, \quad x>0 ; \beta \geq 0, \alpha, \lambda>0
$$

The model selection is carried out using the Akaike information criterion (AIC), the Bayesian information criterion (BIC), the Hannan-Quinn information criterion (HQIC) and the consistent Akaike information criteria (CAIC) defined by:

$$
\begin{aligned}
& \mathrm{AIC}=-2 l(\hat{\boldsymbol{\theta}})+2 q \\
& \mathrm{BIC}=-2 l(\hat{\boldsymbol{\theta}})+q \log (n) \\
& \mathrm{HQIC}=-2 l(\hat{\boldsymbol{\theta}})+2 q \log (\log (n)) \\
& \mathrm{CAIC}=-2 l(\hat{\boldsymbol{\theta}})+\frac{2 q n}{n-q-1}
\end{aligned}
$$

where $l(\hat{\boldsymbol{\theta}})$ denotes the log-likelihood function evaluated at the maximum likelihood estimates for parameters $\theta, q$ is the number of parameters, and $n$ is the sample size.

Table 2 provide the MLEs of the model parameters. The model with minimum AIC (or BIC, CAICand HQIC) value is chosen as the best model to fit the data. From Table 3, 
Table 2 MLEs for bladder cancer data

\begin{tabular}{lccllll}
\hline Distribution & \multicolumn{7}{l}{ MLEs } & & & & \\
\cline { 2 - 7 } & $\hat{\boldsymbol{\lambda}}$ & $\hat{\boldsymbol{\alpha}}$ & $\hat{\boldsymbol{\beta}}$ & $\hat{\boldsymbol{\gamma}}$ & $\hat{\boldsymbol{a}}$ & $\hat{\boldsymbol{b}}$ \\
\hline 1. WLX & 20.8789 & 5.1265 & 1.5857 & - & - & - \\
2. GL & 20.5807 & 4.7541 & - & - & 1.5858 & - \\
3. KWL & 12.2973 & 0.3911 & - & - & 1.5162 & 11.0323 \\
4. TrEL & 0.0546 & 3.3391 & 1.71418 & 0.2440 & - & - \\
5. WL & 1.5794 & 0.2566 & - & - & 2.4215 & 1.8639 \\
6. McL & 11.2929 & 0.8085 & 2.1046 & - & 1.5060 & 4.1886 \\
7. BL & 23.9281 & 3.9191 & - & - & 1.5853 & 0.1572 \\
8. Lomax & 121.0225 & 13.9384 & - & - & - & - \\
9. EPL & 0.00804 & 0.2387 & 59.8378 & - & - & - \\
10. ExL & 0.0800 & 1.0644 & 0.0060 & - & - & - \\
\hline
\end{tabular}

Table 3 The Measures AIC, BIC, HQIC, CAIC for bladder cancer data

\begin{tabular}{llllll}
\hline Distribution & \multicolumn{5}{l}{ Measures } \\
\cline { 2 - 6 } & - Log L & AIC & BIC & HQIC & CAIC \\
\hline 1. WLX & -410.07 & 826.14 & 834.70 & 829.62 & 826.33 \\
2. GL & -410.08 & 826.16 & 834.71 & 829.64 & 826.36 \\
3. KWL & -409.94 & 827.88 & 839.29 & 832.52 & 828.14 \\
4. Tr EL & -410.43 & 828.87 & 840.2 & 833.51 & 829.13 \\
5. WL & -410.81 & 829.62 & 841.03 & 834.26 & 829.88 \\
6. McL & -409.91 & 829.82 & 844.09 & 835.62 & 830.14 \\
7. BL & -411.74 & 831.47 & 842.89 & 836.12 & 831.74 \\
8. Lomax & -413.83 & 831.67 & 837.37 & 833.98 & 831.80 \\
9. EPL & -413.83 & 833.67 & 842.22 & 837.14 & 833.86 \\
10. EXL & -414.98 & 835.96 & 844.51 & 839.43 & 836.15 \\
\hline
\end{tabular}

Table 4 Goodness-of-fit tests for bladder cancer data

\begin{tabular}{lllll}
\hline Distribution & \multicolumn{4}{l}{ Statistics } \\
\cline { 2 - 5 } & $\boldsymbol{W}_{\boldsymbol{n}}^{\mathbf{2}}$ & $\boldsymbol{A}_{\boldsymbol{n}}^{\mathbf{2}}$ & $\boldsymbol{U}_{\boldsymbol{n}}^{\mathbf{2}}$ & $\boldsymbol{L}_{\boldsymbol{n}}$ \\
\hline 1. WLX & 0.026524 & 0.18137 & 31.5282 & 0.47912 \\
2. EL & 0.026820 & 0.18341 & 31.5288 & 0.48320 \\
3. GL & 0.026190 & 0.18089 & 31.5298 & 0.47768 \\
4. TrEL & 0.031438 & 0.22753 & 31.5314 & 0.53413 \\
5. WL & 0.038295 & 0.26273 & 31.5434 & 0.57746 \\
6. BL & 0.025822 & 0.17872 & 31.5272 & 0.47508 \\
7. Lomax & 0.212589 & 1.37456 & 31.7017 & 1.05935 \\
8. EPL & 0.226761 & 1.45110 & 31.7139 & 1.07341 \\
9. EXL & 0.179676 & 1.0908 & 31.6934 & 1.08401 \\
\hline
\end{tabular}

we note that the WLx model gives the lowest values for the AIC, BIC, HQIC and CAIC statistics among all fitted models. So, the WLx model could be chosen as the best model comparable GL, KwL, TrEL, WL, McL, BL, EPL, ExL and Lomax distributions. 
Now, the formal goodness-of-fit tests are applied in order to verify which distribution fits better to these data. The Cramér-von Mises $\left(W_{n}^{2}\right)$, Anderson-Darling $\left(A_{n}^{2}\right)$, Watson $\left(U_{n}^{2}\right)$ and Liao-Shimokawa $\left(L_{n}\right)$ tests statistics are considered. For further details, the reader is refereed to Chen and Balakrishnan (1995). In general, the smaller the values of the $W_{n}^{2}, A_{n}^{2}, U_{n}^{2}$ and $L_{n}$, the better the fit to the data. The values of the statistics $W_{n}^{2}, A_{n}^{2}$, $U_{n}^{2}$ and $L_{n}$ are given in Table 4 . Based on these statistics, the WLx model fits the bladder cancer data better than TrEL, WL, EPL, EXL and Lomax models and gives values close to GL and BL it can be concluded.

\section{Conclusion}

In this paper, WLx distribution is proposed. A mathematical treatment of the proposed distribution including explicit formulas for the density and hazard functions, moments, order statistics have been provided. The estimation of the parameters has been approached by maximum likelihood and method of moments and the observed information matrix is obtained. The usefulness of the new distribution is illustrated in an analysis of Bladder cancer data. The results indicate that the WLx distribution applicable and more flexible than other extensions of Lomax distribution.

Competing interests

The author declares that he has no competing interests.

Received: 27 July 2016 Accepted: 6 October 2016

Published online: 24 October 2016

\section{References}

Abdul-Moniem IB, Abdel-Hameed HF (2012) On exponentiated Lomax distribution. Int J Math Arch 3:2144-2150

Afify AZ, Nofal ZM, Yousof HM, Gebaly YM, Butt NS (2015) The transmuted Weibull Lomax distribution: properties and application. Pak J Stat Oper Res 11:135-152

Al-Zahrani B (2015) An extended Poisson-Lomax distribution. Adv Math Sci J 4(2):79-89

Arnold BC, Balakrishnan N, Nagaraja HN (1992) A first course in order statistics. Wiley, New York

Ashour SK, Eltehiwy MA (2013) Transmuted exponentiated lomax distribution. Aust J Basic Appl Sci 7(7):658-667

Bahadur RR (1964) On Fisher's bound for asymptotic variances. Ann Math Stat 35(4):1545-1552

Bryson MC, Siddique MM (1969) Some criteria for aging. J Am Stat Assoc 64:1472-1483

Chen G, Balakrishnan N (1995) A general purpose approximate goodness-of-fit test. J Qual Technol 27:154-161

Cordeiro GM, Ortega EMM, Popović BV (2015) The gamma-Lomax Distribution. J Stat Comput Simul 85(2):305-319

El-Bassiouny AH, Abdo NF, Shahen HS (2015) Exponential Lomax distribution. Int J Comput Appl 121(13):24-29

Ghitany ME, AL-Awadhi FA, Alkhalfan LA (2007) Marshall-Olkin extended Lomax distribution and its applications to censored data. Commun Stat Theory Methods 36(10):1855-1866

Glaser RE (1980) Bathtub and related failure rate characterizations. J Am Stat Assoc 75:667-672

Gupta RC, Akman O (1995) Mean residual life function for certain types of non-monotonic ageing. Commun Stat Stoch Models 11:219-225

Gupta PL, Gupta RC (1983) On the moments of residual life in reliability and some characterization results. Commun Stat Theory Methods 12(4):449-461

Gupta AK, Tripathi RC (1996) Weighted bivariate logarithmic series distributions. Commun Stat Theory Methods 25:1099-1117

Gupta RC, Ghitany ME, Al-Mutairi DK (2010) Estimation of reliability from Marshall-Olkin extended Lomax distributions. J Stat Comput Simul 80:937-947

Hewa AP (2011) Statistical properties of weighted generalized gamma distribution. Master dissertation, Statesboro, Georgia

Kundu C, Nanda AK (2010) Some reliability properties of the inactivity time. Commun Stat Theory Methods 39:899-911

Leadbetter MR, Lindgren G, Rootzén H (1987) Extremes and related properties of random sequences and processes. Springer, New York

Lee ET, Wang JW (2003) Statistical methods for survival data analysis, 3rd edn. Wiley, NewYork

Lemonte AJ, Cordeiro GM (2013) An extended Lomax distribution. Statistics 47(800-816):2013

Lomax KS (1987) Business failures: another example of the analysis of failure data. J Am Stat Assoc 49:847-852

Nanda AK, Singh H, Misra N, Paul P (2003) Reliability properties of reversed residual lifetime. Commun Stat Theory Methods 32(10):2031-2042 
Nofal ZM, Afify AZ, Yous of HM, Cordeiro, GM (2016) The generalized transmuted-G family of distributions. Commun Stat Theory Methods (accepted)

Oluyede BO, George EO (2002) On stochastic inequalities and comparisons of reliability measures for weighted distributions. Math Probl Eng 8:1-13

Patil GP, Ord JK (1976) On size biased sampling and related form invariant weighted distribution. Indian J Stat 39:48-61

Patil GP, Rao GR (1978) Weighted distributions and size biased sampling with applications to wildlife populations and human families. Biometrics 34:179-189

Rao CR (1965) On discrete distributions arising out of methods of a ascertainment. In: Patil GP (ed) Classical and contagious discrete distributions. Pergamon Press and Statistical Publishing Society, Calcutta, pp 320-332

Rao CR (1985) Weighted distributions arising out of methods of ascertainment. In: Atkinson AC, Fienberg SE (eds) A celebration of statistics. Springer, New York, pp 543-569

Stene J (1981) Probability distributions arising from the ascertainment and the analysis of data on human families and other groups. Stat Distrib Sci Work Appl Phys Soc Life Sci 6:233-244

Tahir MH, Cordeiro GM, Mansoor M, Zubair M (2015) The Weibull Lomax distribution: properties and applications. Hacettepe J Math Stat 44(2):461-480

Zelen M (1974) Problems in cell kinetics and the early detection of disease. In: Proschan F, Sering RJ (eds) Reliability and biometry. SIAM, Philadelphia

Zografos K, Balakrishnan N (2009) On families of beta- and generalized gamma generated distributions and associated inference. Stat Methodol 6:344-362

\section{Submit your manuscript to a SpringerOpen ${ }^{\circ}$ journal and benefit from:}

- Convenient online submission

\section{- Rigorous peer review}

- Immediate publication on acceptance

Open access: articles freely available online

- High visibility within the field

- Retaining the copyright to your article

Submit your next manuscript at $>$ springeropen.com 